\title{
Dynamic Thermo-Physical Characteristics of High Temperature Gaseous Hydrocarbon Fuel Thermal Power Generation for Regeneratively Cooled Hypersonic Propulsion System
}

\author{
Qing $\mathrm{Xu}^{1,2}$, Haowei $\mathrm{Li}^{1,2, *}$, Yaoxun Feng ${ }^{1,2}$, Xiaoning $\mathrm{Li}^{1,2}$, Changming $\operatorname{Lin}^{1,2}$ and Chaoying Zhou ${ }^{3}$ \\ 1 Department of thermal power and power engineering, School of Mechanical and Power Engineering, \\ Guangdong Ocean University, No.1, Haida Road, Zhan Jiang, 524088, P.R.China \\ 2 Southern Marine Science and Engineering Guangdong Laboratory (Zhanjiang) \\ 3 Department of Mechanical Engineering and Automation, Harbin Institute of Technology Shenzhen \\ School, Shenzhen 518055, the People's Republic of China \\ * Correspondence: lhw_hit@163.com
}

\begin{abstract}
The aspirated hypersonic air-breathing propulsion system requires a large amount of power generation, but its special structure makes it impossible to adopt common power generation methods. The high-temperature gaseous hydrocarbon fuel thermal power generation (TPG) system was developed to solve the power generation problem for hypersonic air-breathing propulsion system. But off-design operating conditions of the hypersonic propulsion system results in a more complex process for both propulsion system and the TPG system. To better analyzing the dynamic thermos-physical characteristics of hypersonic airbreathing propulsion system considering thermalmechanical coupling process among cooling/TPG system, a dynamic analytical model was developed, and the dynamic thermos-physical characteristics of TPG system under different offdesign working conditions were conducted. It can be concluded from the analytical results that the dynamic process of thermos-physical characteristics shows a complex trend under the flight Mach number and fuel equivalence ratio off-design working conditions. Such complexity of dynamic characteristics brings difficulties in fuel supply for the propulsion system.
\end{abstract}

Keywords: dynamic thermos-physical characteristics; hydrocarbon fuel; thermal power generation (TPG); thermo-mechanical coupling effect; hypersonic air-breathing propulsion

\section{Introduction}

Hypersonic propulsion system has become research focus due to its high Mach number working characteristics1. Scramjet is a perfect means to achieve the hypersonic flights because of its unparalleled specific thrust performance2. The feasibility of scramjets has already been verified by the HyShot3 and the HyTech4 programs and has therefore become the focus of the research for hypersonic propulsion systems. The hydrogen or liquid hydrocarbon fuel is adopted as main propellant for scramjet nowadays. Owing to the easy acquisition and storage; liquid hydrocarbon fuel has been a promising propellant for scramjets. Furthermore; the feasibility of hydrocarbon fuel has been successfully verified by the X-51A flight tests5. The liquid hydrocarbon fuel has therefore been the focus of recent research.

Due to the unique structure of scramjet, the conventional power generation methods are not suitable for scramjet6. However, owing to the huge power requirement of the electrical systems of hypersonic vehicles in long range hypersonic flights for various facilities and equipment, such as fuel feeding, environment control and radar7, a new power generation method is at an urgent requirement. A new power generation scheme that can meet the scramjet power requirements and avoid quality penalties8, which derives from the expander cycle rocket engine9 and be called high temperature gaseous hydrocarbon fuel TPG method. The TPG method is based on the principle of expansion of high temperature hydrocarbon fuel after regenerative cooling process[10-12]. When hydrocarbon fuel gets gasified13 in cooling channel and cracks into small hydrocarbons14 which 
helps to enhance the fuel TPG capability. The TPG capability of thermal cracked hydrocarbon fuel is studied with analytical model and experiments at different mass flows, temperatures, and expansion ratios15. Furthermore, to fulfil the fuel supply requirement of scramjets, a turbo-pump test system was developed to validate the feasibility of TPG method in scramjet16. Most published literature focuses on characteristics of the hydrocarbon fuel TPG method under high heat flux conditions17, and TPG characteristics improvement methods18. There is few work have been conducted to study the TPG off-design dynamic characteristics, especially considering both TPG system and propulsion system. The published papers mainly concern on TPG capability and propulsion characteristics individually815-19, which are also steady state. As a result, the dynamic characteristics for TPG system considering the influence of propulsion system is worth to be covered.

To study the hydrocarbon fuel dynamic TPG characteristics considering thermal-mechanical coupling effect of propulsion system, the thermal-mechanical coupling effect between TPG system and propulsion system is discussed at first. Then, an analytical model consists of TPG system and hypersonic propulsion system is developed. After that, the dynamic characteristics of the TPG system under different off-design working conditions are simulated and analyzed. At last, the potential problems aroused by dynamic characteristics of TPG system are discussed. The results show that the dynamic process of the outlet fuel temperature and rotation speed of the TPG system coupled with thermal-mechanical coupling effect is complex, of which there are positive and negative overshoot. Furthermore, the amplitude of overshoot changes a lot, varying with the off-design conditions. However, the dynamic process of fuel temperature and rotation speed causes over-temperature and fuel mass flow control risk for the scramjet.

\section{Analysis on the cooling-power generation thermal coupling process for hypersonic propulsion system}

Based on working process of hypersonic propulsion system and TPG system, hydrocarbon fuel is both adopted as propellant, coolant and working fluid of TPG system. Since the regenerative cooling process is accomplished before TPG of hydrocarbon fuel, high temperature hydrocarbon fuel power generation capability can be largely influenced by the regenerative cooling process. Whilst, the heat source for high temperature hydrocarbon fuel is conducted through combustor, which generated by combusted hydrocarbon fuel. As a result, there is strong thermo-mechanical coupling effect between cooling/combustion process and TPG process. Furthermore, the thermo-mechanical coupling effect becomes complex during different kinds of off-design working conditions.

According to working status of the propulsion system, off-design working conditions of the cooling system can be generalized and divided into two categories, which are heat flux off-design working condition and fuel mass flow off-design working condition. The heat flux off-design working condition leads to variation of fuel temperature. Both the mass flow and heat flux off-design conditions can also be divided into increase and decrease situation. Despite the type of off-design working conditions, there will be positive feedback process, which may lead to the invalid of power generation system and regenerative cooling system. It is worth to be noticed that heat flux of the cooling channel is highly related with flight Mach number and fuel mass flow. As a result, the TPG dynamic thermophysical capability is highly related and becomes more complex due to the thermalmechanical coupling effect between combustion and regenerative cooling process of the hypersonic air-breathing propulsion system. Thermo-mechanical coupling process of off-design working conditions is shown in Figure 1. 


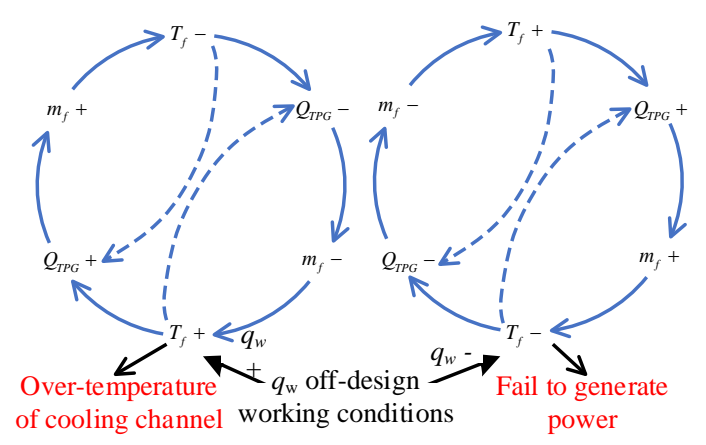

a)

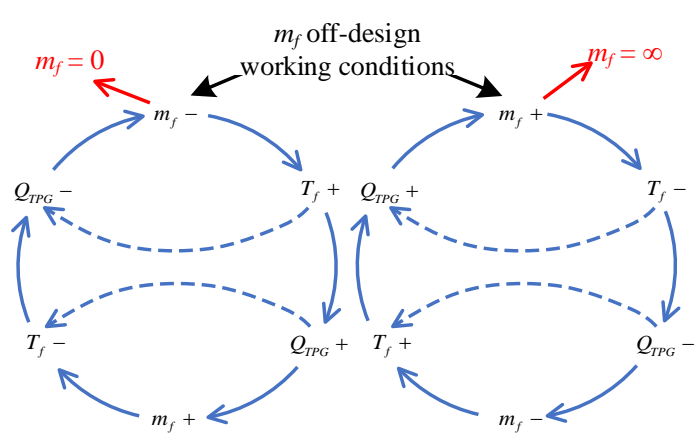

b)

Figure 1 Thermo-mechanical coupling process of the high temperature gaseous hydrocarbon fuel TPG system under different off-design working conditions.

\section{Dynamic analytical model of high temperature gaseous hydrocarbon fuel TPG system considering cooling-power generation thermo-mechanical coupling effect}

High temperature gaseous hydrocarbon fuel TPG process is highly related with regenerative cooling process, while there is strong thermos-mechanical coupling effect between combustion and regenerative cooling process. The hydrocarbon fuel of TPG system is heated in the cooling channel at first. Then, the high temperature gaseous hydrocarbon fuel expands in turbine, and the expansion power is generated by generator driven by the turbine. At last, the expanded high temperature gaseous hydrocarbon fuel is injected into combustor and combusted. Heat flux of the cooling channel is conducted from combustor generated by the combusted hydrocarbon fuel, which is related with working status of hypersonic propulsion system, such as flight Mach number and fuel equivalence ratio. As a result, flight Mach number and fuel equivalence ratio will influence dynamic thermosphysical characteristics of the TPG system during the off-design working conditions. It is worth to notice that the turbine and pump are mounted in the same axis. As a result, turbine and pump share the same rotation speed, which is called turbo-pump rotation speed. To better study the dynamic thermos-physical characteristics of the high temperature gaseous hydrocarbon fuel TPG system, in which the cooling-power generation thermo-mechanical coupling effect is considered. The whole high temperature gaseous hydrocarbon fuel TPG system and its simplified model structure are shown in Figure 2.

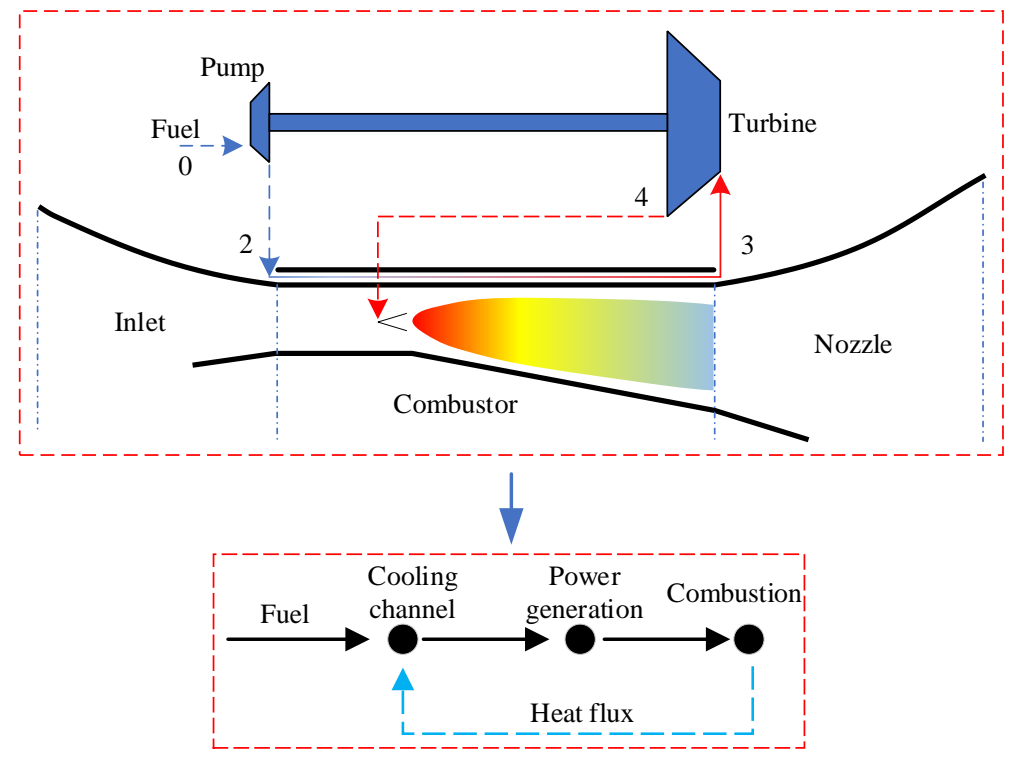

Figure 2 Simplified high temperature gaseous hydrocarbon fuel TPG structure considering coolingpower generation thermo-mechanical coupling effect. 


\subsection{Dynamic model of heat transfer process of the cooling channel}

For zero-dimensional model, the radial flow and heat transfer of the hydrocarbon fuel in cooling channel has not been taken into consideration. Due to the influence of viscous force, velocity of the viscous bottom fuel near the wall of the cooling channel is relatively slow. As a result, the heat transfer process in this part of the fluid is more inclined to be heat conduction than convective heat transfer, which makes the dynamic heat transfer time of this part of hydrocarbon fuel increase. While fuel velocity of the near-central flow is faster, and the heat transfer of this part of fuel tends to be enhanced convective heat transfer, which leads to the decrease of dynamic heat transfer time. Therefore, in the zero-dimensional modeling of cooling channel, the traditional fluid-wall convective heat transfer formula combined with the viscous bottom heat conduction caused by the hydrocarbon fuel flow velocity will be modified, which is expressed as follows: hydrocarbon fuel in the cooling channel is assumed to be two layers, the temperature and heat flux are uniformly distributed in the flow direction. Heat exchange process between wall of cooling channel and the first layer of fluid is considered as convective heat transfer, while the heat transfer process in the first layer of fluid is heat conduction. The first layer of fluid and the second layer of fluid are treated as convective heat transfer. The simplified structure and heat transfer process are shown in Figure 3.

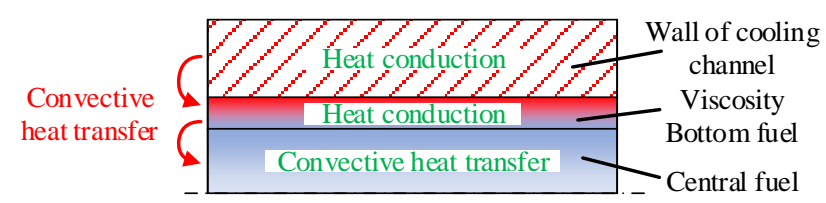

Figure 3 Heat transfer process between cooling channel and hydrocarbon fuel.

The convective heat transfer formula between hydrocarbon fuel and cooling channel wall is written as follows:

$$
Q_{f}=\alpha A_{h}\left(T_{w}-T_{f}^{\prime}\right)
$$

Thermal conductivity in the viscous bottom layer of fuel is expressed as follow:

$$
Q_{f}=A_{h} \frac{\lambda_{f}}{D_{c} / 2}\left(T_{f}^{\prime}-T_{f}\right)
$$

Because of Eq. (1) and Eq. (2), the modified heat transfer can be written as:

$$
Q_{f}=\alpha^{\prime} A_{h}\left(T_{w}-T_{f}\right)
$$

Among them, the original convective heat transfer coefficient $\alpha$ has been modified as a parameter $\alpha^{\prime}$ related to the hydrocarbon fuel thermal conductivity and cooling channel diameter $D_{c}$ :

$$
\alpha^{\prime}=\alpha \cdot \frac{2 \lambda / D_{c}}{2 \lambda / D_{c}+\alpha}
$$

Since the dynamic process of the TPG process is dominated by the heat transfer process of the cooling channel in the high-temperature gaseous hydrocarbon fuel TPG system, and the dynamic time is about $10^{1} \mathrm{~s}$. As a result, the heat transfer process of the cooling channel is expressed as zerodimensional ordinary differential equation, during which the thermal storage time of the hydrocarbon fuel is considered. The heat transfer process of the cooling channel can be expressed as follows:

$$
\rho_{f} c_{p f} V_{f} \frac{d T_{f}}{d t}=Q_{f}
$$

While the heat storage of cooling channel wall process is written as follows:

$$
M_{w} c_{p w} \frac{d T_{w}}{d t}=Q_{w}
$$

Hydrocarbon fuel heat storage and cooling channel heat storage meet energy conservation equation, which is written as follows: 


$$
Q=Q_{f}+Q_{w}
$$

Pressure drop along the cooling channel can be calculated as follows:

$$
p_{p}-p_{b}=f \frac{\rho L u^{2}}{2 D_{c}}
$$

\subsection{Heat flux conducted from the combustor generated by the hydrocarbon fuel}

The air velocity inside the hypersonic propulsion is very high, which is usually over Ma1. As a result, the air dwell time, which is less than $1 \mathrm{~ms}$, is far less than the dynamic heat transfer time of hydrocarbon fuel in the cooling channel. As a result, the combustion of hydrocarbon fuel in the combustor can be treated as steady state compared with the heat transfer in the cooling channel.

It is considered that the total heat absorption $Q$ of the cooling channel is product of the conducted heat flux and heat transfer area between the combustor and the cooling channel. Heat flux of the cooling channel depends on scramjet flight Mach number and the combustion related fuel equivalence ratio. Therefore, there is strong thermal coupling relationship between the total heat absorption $Q$ of the cooling channel and the fuel mass flow, where the expression of the total heat absorption $Q$ can be written as follows:

$$
Q=q_{h} A_{h, c}
$$

Among them, $q_{h}$ is the heat flux of cooling channel conducted from the combustor wall. For a stamped scramjet, given the Mach number, combined with the fuel flow, the total gas temperature can be calculated using the first law of chemical thermodynamics, and the static temperature of the gas can be calculated using the relationship between the total temperature of the runner in the scramjet and the Mach number. The reference enthalpy method can be used to calculate the heat flow on the wall of the combustion chamber, which is written as follows:

$$
q_{h}=S t^{*} \rho^{*} V_{e}\left(h_{a w}-h_{w}\right)
$$

For laminar flow, the relevant Stanton number St* can be calculated as follows:

$$
S t^{*}=\frac{0.332}{\left(\operatorname{Pr}^{*}\right)^{\frac{2}{3}}\left(\operatorname{Re}^{*}\right)^{\frac{1}{2}}}
$$

For turbulence flow, the relevant Stanton number $\mathrm{St}^{*}$ can be calculated as follows:

$$
S t^{*}=\frac{0.0287}{\left(\operatorname{Pr}^{*}\right)^{\frac{2}{5}}\left(\operatorname{Re}^{*}\right)^{\frac{1}{5}}}
$$

The specific enthalpy haw under adiabatic wall temperature can be regarded as the total specific enthalpy of the air in combustor, and hw is the specific enthalpy of the air under the real combustor wall temperature.

When calculating the specific enthalpy under different temperature (adiabatic wall temperature and real wall temperature), the following assumptions are made, namely:

1) The hydrocarbon fuel is completely burned, and its released reaction heat is completely

used to heat the air in the combustor.

2) Fuel mass flow is determined by the fuel equivalence ratio.

Therefore, the specific enthalpy under different temperature can be calculated as follows: 


$$
\begin{gathered}
h_{a w}=m_{c o m b, f} h_{f}-\frac{1}{2} \rho_{e} V_{e}^{2} \\
=m_{c o m b, f} h_{f}-\frac{1}{2} \kappa p_{e} M a_{c o m b}{ }^{2} \\
h_{w}=h\left(T_{w}\right)
\end{gathered}
$$

\subsection{Dynamic model of turbo-pump rotation process}

Considering that the dynamic time of the rotor composed of the turbine, pump and generator is quite close to the dynamin heat transfer time of cooling channel, the dynamic process of the rotor rotation will also be considered. The rotor dynamics equation is expressed as follows:

$$
J_{R} \frac{d \omega}{d t}+f_{R} \omega=\frac{Q_{T u r}-Q_{\text {pump }}-Q_{\text {Gen }}}{\omega}
$$

Where, $Q_{T u r}$ is the total thermal expansion work of the high temperature gaseous hydrocarbon fuel, $Q_{p u m p}$ is the required pump compress power and $Q_{G e n}$ is the power generated by generator. Fuel mass flow for the cooling channel and TPG system is decided by combustion of the propulsion system. As a result, the total thermal expansion power, pump power and generator power are calculated as follows.

$$
\begin{gathered}
Q_{T u r}=m_{f} \Delta h_{s} \\
Q_{p u m p}=\frac{m_{f} p_{p}}{\rho_{0}} \\
Q_{G e n}=N_{G e n} \omega \\
T_{G e n}=\Theta \omega \\
\Theta=\frac{P}{2 \pi} \frac{Z_{a v} B_{a v}{ }^{2} l_{a v}{ }^{2} r}{R_{a}}
\end{gathered}
$$

It is worth to be mentioned that $Q_{G e n}$ is the generated power, which is high related with the rotation speed. As a result, the rotation speed can be used to characterize the power generation status.

The high-temperature gaseous hydrocarbon fuel TPG performance evaluation model for the propulsion system under variable operating conditions is derived from the previous work on combustor model coupled with regenerative cooling process20. In addition, the high-temperature gaseous hydrocarbon fuel expansion type is added, which is developed in the previous work1518.The principle and calculation logic of the model are shown in Figure 4.



Figure 4 Calculation strategy of the high temperature hydrocarbon fuel TPG capability.

\section{Dynamic characteristics simulation of hypersonic propulsion gaseous hydrocarbon fuel TPG system}

As discussed above, the heat flux cooling channel and fuel mass flow will have a great impact on dynamic thermos-physical characteristics, which is mainly determined by the flight Mach number 
and fuel equivalent ratio. Therefore, the dynamics of the thermodynamic power generation system will be explored through the model under the flight Mach number and fuel equivalence ratio offdesign working conditions. The basic working condition points shown in the table are given, which can be compared with the off-design working conditions. Variable operating conditions include the following: 1) Mach number increases and decreases step changes; 2) Mach number changes with aircraft acceleration; 3) Fuel equivalence ratio increases and decreases. Error! Reference source not found. shows the values of the reference points during the system's changing working conditions.

Table 1. Basic working condition of flight Mach number and fuel equivalence ratio.

\begin{tabular}{ccc}
\hline Basic working condition & Flight Mach number & Fuel equivalence ratio \\
\hline Value & 6.5 & 0.8 \\
\hline
\end{tabular}

\subsection{Flight Mach number off-design conditions}

When the flight Mach number increases, outlet fuel temperature of the cooling channel tends to increase. Furthermore, the dynamic process of fuel temperature and rotor speed shows complicated characteristics. Fuel temperature increases with flight Mach number step increase, which is also accompanied with positive overshoot. With increase of the flight Mach number, the temperature of the combusted air relatively increases, which in turn increases heat flux of the cooling channel. It is obvious that the larger the Mach number increase, the higher the heat flux of the cooling channel. As a result, the fuel temperature is correspondingly higher. According to the results shown in Figure 5, the dynamic time of fuel temperature is about 20 30s.

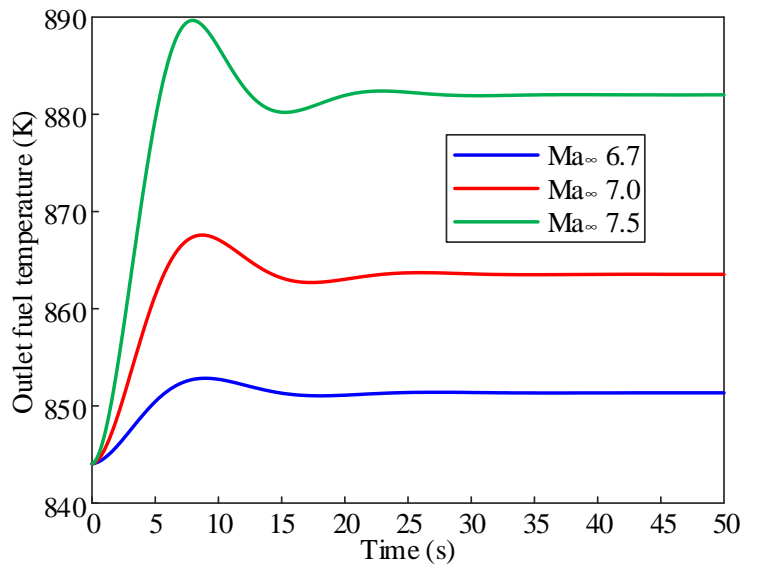

Figure 5 Dynamic process of the outlet fuel temperature under flight Mach number step off-design conditions.

Rotation speed of the turbo-pump generally increases with step increase of the flight Mach number, but the dynamic process is relatively complicated, which decreases with negative overshoot at first and then increases with positive overshoot. Results are shown in Figure 6. As the flight Mach number step increases, the fuel mass flow required for combustion decreases and the fuel temperature gradually increases. Therefore, the fuel thermal expansion capability increases. However, the increase of fuel thermal expansion capability aroused by fuel temperature cannot compensate for the decrease in fuel flow and weaken the fuel thermal expansion capability, which leads to negative overshoot of rotation speed. After that, fuel temperature gradually increases, the fuel thermal expansion capability increases, and the rotation speed of turbo pump increase, during which, there is positive overshoot of rotation speed. The dynamic time of the turbo-pump speed is similar with that of the fuel temperature, which is about 20 30s. 


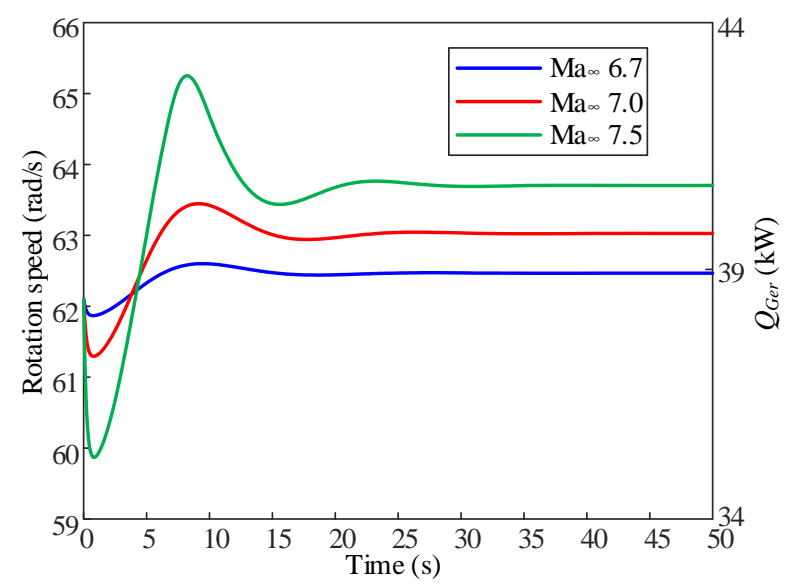

Figure 6 Dynamic process of the rotation speed under flight Mach number step off-design conditions.

\subsection{Acceleration off-design conditions}

During the scramjet normal working conditions, the flight Mach number increases gradually with the acceleration of the aircraft. Therefore, the dynamic response characteristics of the thermodynamic power generation system will be discussed when the flight Mach number changes with given acceleration. In order to discuss the influence of acceleration on cooling and expansion TPG system characteristics, the total increase of flight Mach number is 0.2, while the acceleration varies from $0.08 \mathrm{~g}$ to $\infty$ (which is also considered as Mach number step variation). As a result, dynamic characteristics of the outlet fuel temperature for the cooling channel and the turbo-pump rotation speed show the same trends with flight Mach number step change conditions. When the acceleration increases, the outlet fuel temperature increases, and the positive overshoot of fuel temperature is larger, which is shown in Figure 7. The reasons at acceleration step change conditions are the same with flight Mach number step change conditions. What is different is that while the acceleration is larger, the dynamic time of outlet fuel temperature is shorter. That is because the larger acceleration causes shorter Mach number increasing time when the Mach number increasing amount is constant. Although larger acceleration provides shorter dynamic time, it causes positive overshoot of fuel temperature, which may lead to over temperature risk of combustor wall.



Figure 7 Dynamic process of the outlet fuel temperature under different acceleration off-design conditions.

The rotation speed of turbo-pump dynamic process is just like that of the outlet fuel temperature, it is also that larger acceleration, the shorter dynamic time is, which is shown in Figure 8. The acceleration of a ram/super-combustion ramjet scramjet will not be particularly large. According to 
$\mathrm{X}-51 \mathrm{~A}$ flight data, its acceleration is $0.18 \mathrm{~g}$. Therefore, during the normal operation of a ram/supercombustion ram scramjet, the fuel temperature and the speed of the turbo pump should not change significantly. However, there may be a step change of acceleration in emergencies, for example, unstart/restart status. In this case, the positive/negative overshoot of the fuel temperature and the turbo pump speed will be quite large, which may harm scramjet safety.

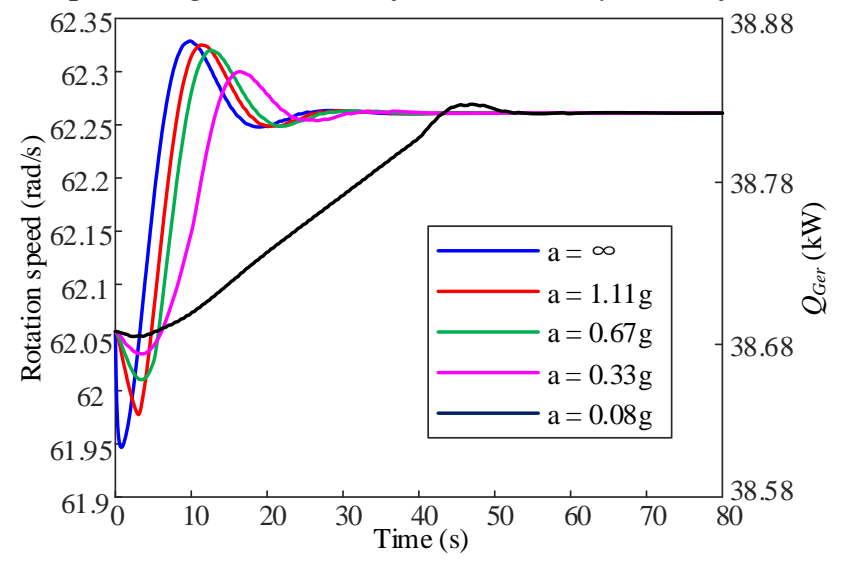

Figure 8 Dynamic process of the rotation speed under different acceleration off-design conditions.

\subsection{Fuel equivalence ratio off-design conditions}

To study dynamic characteristics of TPG system at the fuel mass flow variation condition during the scramjet working process, dynamic simulation of TPG system under fuel equivalence ratio offdesign condition is conducted, in which the fuel equivalence ratio step changed. When the fuel equivalence ratio step decreases, outlet fuel temperature of the cooling channel increases. Meanwhile, and there is a slight positive overshoot during the increasing process. When the fuel equivalence ratio decrease amplitude is larger, the fuel temperature increases higher, and the overshoot amplitude shows an increasing trend. While the fuel equivalence ratio decreases, the fuel temperature decreases, and a negative overshoot occurs, which are shown in Figure 9. When the fuel equivalence ratio is increased, the combustion in the combustion chamber is enhanced, and the wall heat flow shows an increasing trend, but the fuel flow in the cooling channel increases, the heat sink increases, and the increase in heat sink dominates, so the fuel temperature shows a downward trend. Conversely, the fuel equivalence ratio reduces the heat sink of the fuel, and the decrease in the total heat flux of the cooling channel is smaller than the heat sink of the fuel, resulting in an increase in fuel temperature. The change of the turbo-pump rotation speed with the equivalence ratio is opposite to the fuel temperature. The rotation speed decreases with the decrease of the fuel equivalence ratio and increases with the increase of the equivalence ratio, and there are also positive and negative overshoots during the fuel equivalent step off-design conditions. Although the increase of the equivalence ratio leads to a decrease in fuel temperature, the increase in fuel flow increases the fuel TPG capability. As a result, the turbo-pump rotation speed increases, which are shown in Figure 10. On the contrary, decrease of the fuel equivalence ratio weakens the fuel TPG capability, the turbo pump speed tends to decrease. The dynamic time of outlet fuel temperature for the cooling channel is about $10-30$ s. 


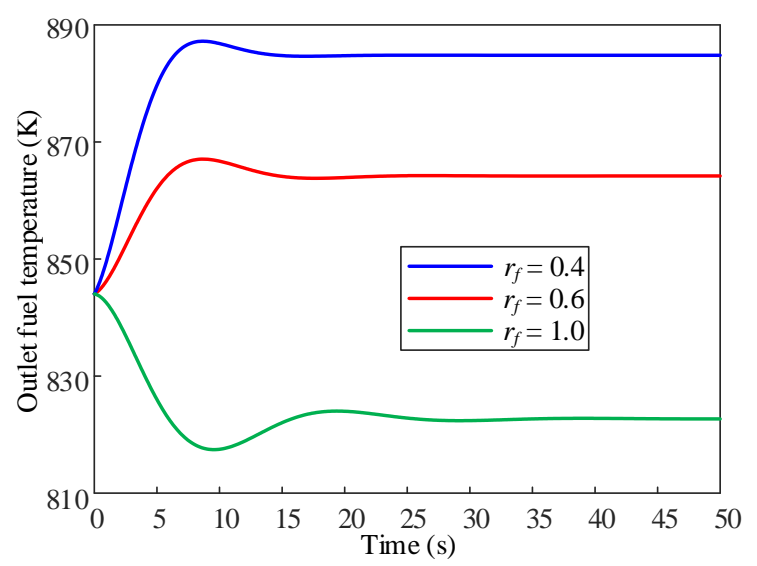

Figure 9 Dynamic process of the outlet fuel temperature under different fuel equivalence ratio offdesign conditions.

While the dynamic time of the fuel equivalence ratio step decrease off-design condition is longer than that of the increase step off-design condition. Furthermore, the dynamic time of both fuel temperature and rotation speed is shorter when the decrease of fuel equivalence ratio is larger. When the equivalence ratio decreases, the fuel temperature increases, the fuel flow velocity in the cooling channel increases, and the degree of turbulence increases, which greatly improves the convective heat transfer coefficient of the fuel and enables the fuel to reach a thermal equilibrium state faster. However, fuel heat transfer dynamic time tends to be longer under fuel equivalence ratio increase condition. Due to the small moment of inertia for the rotor used in the high-temperature gaseous hydrocarbon fuel TPG system, which is generally 10-2 to 10-1 ( $\mathrm{kg} \mathrm{m} 2)$, the dynamic response time of the speed is 101s, which is related to the heat transfer The time scale is similar, so there is a certain degree of coupling effect between rotor rotation and heat transfer, which may bring certain difficulties to control of the turbo-pump outlet fuel mass flow.

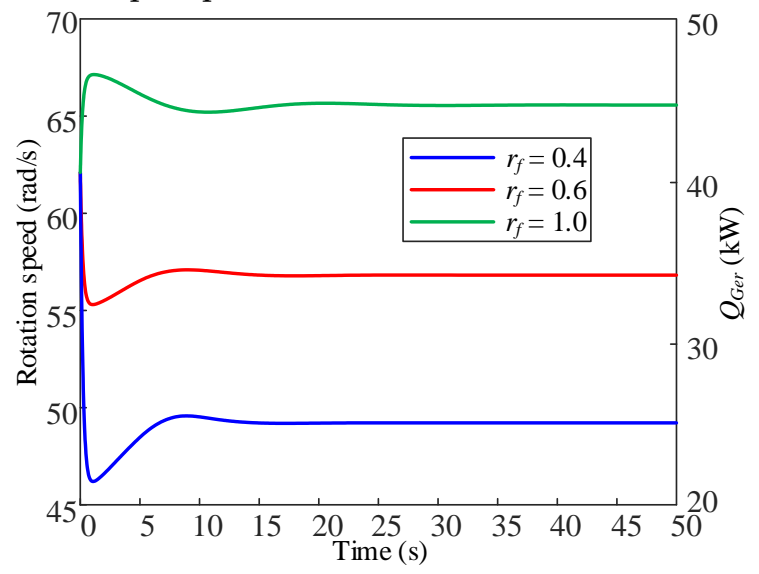

Figure 10 Dynamic process of the rotation speed under different fuel equivalence ratio off-design conditions.

4.4. Influence of dynamic thermo-physical characteristics on fuel mass flow for high temperature hydrocarbon fuel TPG system

Based on the above-mentioned dynamic process, the characteristics of the turbo pump speed are discussed to discuss the dynamic changes of the fuel flow and pressure at the pump outlet with the turbo pump speed. Among them, take the Mach number step change from Ma 6.7 to Ma 7.0. The pumps used in aerospace propulsion systems are mainly divided into two categories: positive displacement pumps and centrifugal pumps. Among them, the displacement pump has a fixed displacement per revolution, so the pump outlet flow rate is proportional to the speed. The centrifugal pump relies on a high-speed rotating impeller to compress the hydrocarbon fuel, which 
increases the pressure and kinetic energy of the fluid. The centrifugal pump outlet pressure is related to its speed. Generally speaking, volumetric pumps are mostly used as a constant flow fuel supply source, while centrifugal pumps are mostly used as a constant pressure supply source. It is assumed that the positive displacement pump is in an ideal state, and the leakage amount is 0 . The relationship between the outlet flow rate and the rotational speed is shown in equation (5-1).

$$
m_{c v p}=\frac{V_{c v p} \omega_{c v p} \rho_{0}}{120 \pi}
$$

Assume that the installation angle of the outlet blade of the centrifugal pump is $90^{\circ}$, and the total outlet head of the centrifugal pump is only proportional to the square of the rotational speed.

$$
\begin{gathered}
H_{c e p}=\frac{1}{g} u_{c e p}{ }^{2} \\
u_{c e p}=0.5 D_{c e p} \omega_{c e p} \\
p_{c e p}=0.25 \rho_{0} D_{c e p}{ }^{2} \omega_{c e p}^{2}
\end{gathered}
$$

Combining the dynamic process of turbo-pump speed with Mach number and fuel equivalence ratio step change, we can get the dynamic change process of the volumetric pump outlet fuel flow and centrifugal pump outlet pressure can be obtained under different conditions. To conveniently indicate the relative changes in flow and pressure, the flow and pressure at the initial moment are dynamically calculated as the "0" state point, and the relative changes in flow and pressure are the flow and pressure at any time and the "0" state point. ratio. It can be seen from the results that under the condition that the Mach number increases step by step, the relative change rate of the fuel flow at the exit of the positive displacement pump shows a trend of first negative adjustment and then overshoot, and the final relative flow is greater than 1 . The specific dynamic process is shown in Figure 11. In other words, the pump outlet flow rate first decreases and then increases with the pump speed, and the final fuel flow rate is greater than the "0" state fuel flow rate.

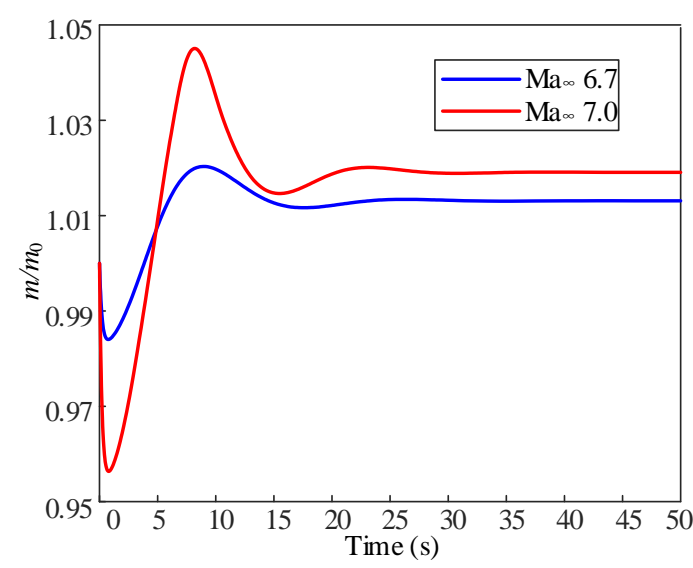

a)

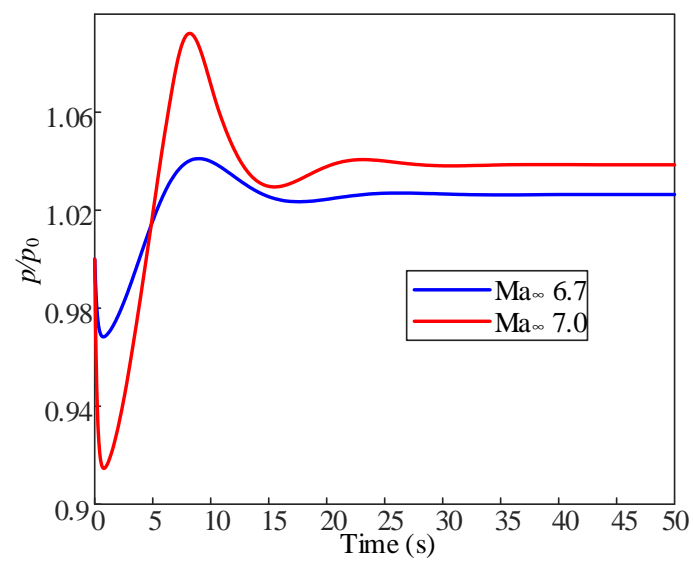

b)

Figure 11 Relevant fuel mass flow and pump pressure dynamic characteristics under flight Mach number off-design working conditions.

And the centrifugal pump outlet pressure changes with the flow. Under the condition that the fuel equivalence ratio increases step by step, there is an overshoot in the dynamic change process of the relative change rate of the fuel flow at the displacement pump and the fuel pressure at the centrifugal pump outlet. The specific dynamic processes are shown in Figure 12. 


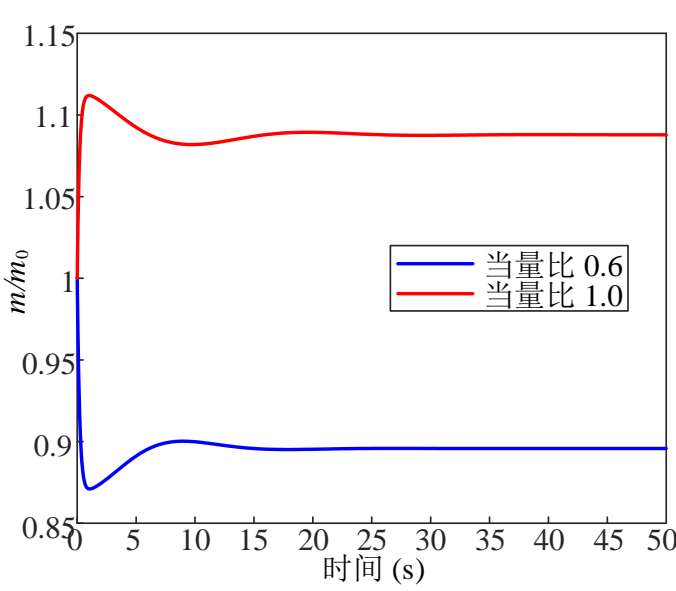

a)



b)

Figure 12 Relevant fuel mass flow and pump pressure dynamic characteristics under fuel equivalence ratio off-design working conditions.

In the previous section, under the decoupling condition of the fuel flow at the turbo-pump outlet and the required flow in the combustion chamber, the dynamics of the fuel temperature at the outlet of the cooling channel and the speed of the turbine pump under two different scramjet operating conditions, such as fuel equivalence ratio and Mach step change, were explored response process. The results show that the dynamic response process of the turbo pump speed during the abovementioned two scramjet changing conditions is more complicated, and there is a certain degree of overshoot or undershoot. And this dynamic characteristic brings certain difficulties to the control of fuel flow. For an scramjet, the fuel flow depends on the scramjet's needs. In other words, the incoming air flow captured by the scramjet and the expected operating state of the scramjet determine the flow required for scramjet combustion. It is difficult for the turbo-pump output fuel flow to be selfbalanced with the scramjet combustion required flow rate without adding additional control means, so the turbopump output fuel flow rate needs to be controlled. We want to use high-temperature hydrocarbon fuel for thermodynamic power generation as much as possible while ensuring the normal performance of the scramjet. For generators, increasing the speed is conducive to increasing power generation. However, because the turbo pump is coaxial with the high-temperature gaseous hydrocarbon fuel TPG system, if the outlet fuel control is achieved by adjusting the turbo pump speed, it will inevitably lead to loss of power generation. It is important to adjust the structure of the pump to obtain the required flow during the operation of the turbo pump. Therefore, fuel flow control can only be achieved by diverting or throttling the fuel at the pump outlet.

\section{Conclusions}

To further study the thermos-physical dynamic characteristics of high temperature gaseous hydrocarbon fuel TPG system considering thermal-mechanical coupling effect. Based on that, an analytical model consists of TPG system and hypersonic propulsion system is developed. After that, the dynamic characteristics of the TPG system under different off-design working conditions are simulated and analyzed. Several results have been concluded as follow.

1 In flight Mach number step change off-design condition, the outlet fuel temperature increases with the increase of flight Mach number. As a result, the rotation speed shows the increase trends. Both dynamic time of outlet fuel temperature and rotation speed are 20-30s. The dynamic process of outlet fuel temperature and rotation speed shows complex characteristics. There is positive overshoot of outlet fuel temperature during the flight Mach number step increase off-design process, which may cause over-temperature risk of combustor. Whilst, the dynamic process of rotation speed decreases with negative overshoot at first, then increases with positive overshoot.

2 In flight Mach number increases with acceleration off-design condition, the dynamic process of outlet fuel temperature and rotation speed shows the same trend with that of the flight 
Mach number step off-design condition. The different is that the acceleration is larger, the dynamic time is shorter. However, the overshoot amplitude is larger, which may lead to over-temperature risk of combustor.

3 In fuel equivalence ratio step off-design condition, the outlet fuel temperature increases with the decrease of fuel equivalence ratio, which couples with positive overshoot. While the rotation speed decreases with the decrease of fuel equivalence ratio, which couples with negative overshoot. The dynamic time of outlet fuel temperature and rotation speed is about 10-30s.

4 According to the difference of pump structure, the fuel supply principle is different. For volumetric pumps are mostly used as a constant flow fuel supply source, while centrifugal pumps are mostly used as a constant pressure supply source. The dynamic process of rotation speed causes the outlet fuel mass flow and pressure changes according the pump structure, which leads to the unstable of the fuel mass flow for the scramjet.

Author Contributions: Conceptualization, Q. X. and H. L.; formal analysis, Y. F.; data curation, X. L.; writing original draft, Q. X. and H. L.; writing - review \& editing, Q. X. and C. Z.

Funding: This research work is supported by the Fund of Southern Marine Science and Engineering Guangdong Laboratory (ZJW-2019-01), the Natural Science Foundation of Guangdong Province of China (No. 2019A1515011551) and the Project of Fundamental Research of Shenzhen Science and Technology Plan(No. JCYJ20170307151117299).

Acknowledgments: The authors thank the reviewers for their valuable advice on this paper. Furthermore, the best gratitude and wishes are dedicated to the Energies' staff and editors for handling the paper.

Conflicts of Interest: The authors declare no conflicts of interest.

\section{References}

1. Fry, R.S. A century of ramjet propulsion technology evolution. J. Propul. Power. 2004; 20(1): 27-58.

2. Cui, T.; Ou Y. Modeling of scramjet combustors based on model migration and process similarity. Energies. 2019; 12(13): 2516.

3. Hass, N.; Smart, M.; Paull, A. Flight data analysis of the HYSHOT 2. AIAA Paper 2005-3354, May 2005.

4. Mercier, R.; Ronald, T. Hypersonic technology (HyTech) program overview. AIAA Paper 1998-1566, April 1998.

5. Joseph, M.H.; James, S.M., Richard C.M. The X-51A scramjet flight demonstration program. AIAA Paper 2008-2540, April 2008.

6. Cheng, K.L.; Qin, J.; Sun, H.C.; Dang, C.L.; Zhang, S.L.; Bao, W. Performance comparison on wall cooling and heat supply for power generation between fuel- and liquid metal-cooled scramjet. Aerosp. Sci. Technol. 2019; 93: 105294.

7. Lou, F.; Fabian, J.C.; Key, N.L. Experimental investigation of flow distortion in an auxiliary-power-unitstyle inlet system. J. Propul. Power. 2016; 32(4): 892-902.

8. Zhang, D.; Qin, J.; Feng, Y.; Ren, F.Z.; Bao, W. Performance evaluation of power generation system with fuel vapor turbine onboard hydrocarbon fueled scramjets. Energy. 2014; 77: 732-741.

9. Manski, D.; Goertz, C.; Saoslash, H.; Sassnick, H.D.; Hulka, J.R.; Goracke, B.D.; Levack, D.J.H. Cycles for earth-to-orbit propulsion. J. Propul. Power. 2012; 14(5): 588-604.

10. Wang, Y.H; Li, S.F.; Dong, M. Numerical study on heat transfer deterioration of supercritical n-Decane in horizontal circular tubes. Energies. 2014; 7(11): 7535-7554.

11. Dong, Y.W.; Wang, E.T.; You, Y.C.; Yin, C.P.; Wu, Z.P. Thermal protection system and thermal management for combined-cycle engine: review and prospects. Energies. 2019; 12(2): 240.

12. Cheng, K.L.; Feng, Y.; Lv, C.W.; Zhang, S.L.; Qin, J.; Bao, W. Performance evaluation of waste heat recovery systems based on semiconductor thermoelectric generators for hypersonic vehicles. Energies. 2017; 10(4): 570.

13. Sun, X.; Meng, H.; Zheng, Y. Asymmetric heating and buoyancy effects on heat transfer of hydrocarbon fuel in a horizontal square channel at supercritical pressures. Aerosp. Sci. Technol. 2019; 93: 105358. 
14. Feng, Y.; Cao, Y.; Liu, S.Y.; Qin, J.; Hemeda, A.A.; Ma, Y.B. The influence of coking on heat transfer in turbulent reacting flow of supercritical hydrocarbon fuels. Int. J. Haet Mass Tran. 2019; 144: 118623.

15. Li, H.W.; Qin, J.; Jiang, Y.G.; Zhang, D.; Cheng, K.L.; Bao, W.; Huang, H.Y. Experimental and theoretical investigation of power generation scheme driven by thermal cracked gaseous hydrocarbon fuel for hypersonic vehicle. Energ. Convers. Manage. 2018; 165: 334-343.

16. Wu, X.Y.; Yang, J.; Zhang, H.; Shen, C.B. System design and analysis of hydrocarbon scramjet with regeneration cooling and expansion cycle. J. Therm. Sci. 2015; 24(4): 350-355.

17. Zhang, H.; Shen, C.B.; Wu, X.Y.; Tan, J.G.; Xi, W.X. Design and optimization of hydrocarbon-fueled scramjet start-up scheme with expansion cycle. 62nd International Astronautical Congress. IAC-11-C4.5.8, 2011.

18. Li, H.W.; Qin, J.; Bao, W.; Huang, H.Y. Performance improvement of gaseous hydrocarbon fuel driven thermal power generation systems for hypersonic vehicles. Energ. Convers. Manage. 2019; 199: 111949.

19. Wu, X.Y.; Yang, J.; Jin, X. Experimental study of operation performance for hydrocarbon fuel pump with low specific speed. J. Therm. Sci. 2017; 26(5): 440-447.

20. Zhang, D.; Feng, Y.; Zhang, S.; Qin, J.; Cheng, K.; Bao, W.; Yu, D. Quasi-one-dimensional model of scramjet combustor coupled with regenerative cooling. J. Propul. Power. 2016; 32: 687-697. 